\title{
Driver's eye detection and state analysis in fatigue driving
}

\author{
Sun Guangmin ${ }^{1, a}$, Jia Linan ${ }^{1, b}$, Zheng Kun ${ }^{1, c}$ \\ ${ }^{1}$ Department of Electronic Engineering, Beijing University of Technology, Beijing, 100124, China \\ agmsun@bjut.edu.cn, ${ }^{b}$ jialinan1990@163.com, czhengkun@bjut.edu.cn
}

Keywords: Image preprocessing, Face region segmentation, Eye detection, Ellipse fitting

\begin{abstract}
As one of the most important features of human face, the eye location plays a fundamental role in many application fields. For example, by judging the eyes state, defining whether the driver is in the state of fatigue. At present, most of the eye detection algorithms in fatigue driving are based on laboratory simulation environment. Therefore, this paper is based on the real car environment, collecting the pictures from the driver simulating the driving condition. Then, using the method of region segmentation and ellipse fitting to locate and analyze the state of eyes. All experiment pictures are collected by Android phone front camera. The algorithm runs fast and is suitable for real-time processing. At the same time, there is a good performance of eye location in the situation where there exists a certain facial orientation angle.
\end{abstract}

\section{Introduction}

Fatigue driving is one of the main causes leading to traffic accidents.[1] According to the video image to detect the driver's facial features, so as to make fatigue detection has become a hot research. The open state of the driver's eyes can reflect the fatigue degree directly, so it can be used to judge the driver's fatigue by using image processing method. Conventional eye location algorithm include: gray projection, edge extraction, template matching, etc. Such as Sun et al. [2] using edge detection method to locate the human eye, the method needs preprocessing and cannot meet the real-time requirements. Phimoltares et al. [3] using deformable template to locate the human eye, the method is simple, but the calculation is large. In addition, Huang Kun [4] using the improved Adaboost algorithm for face and eye location, however, the algorithm is limited to the training of the face classifier, the accuracy rate decreased when face pose changed.

Eye state recognition methods are mainly based on machine learning classification methods and image analysis methods. Such as Zhang and so on [5]using RBF neural network for eye state recognition, but it needs a large number of eye template for training and the amount of calculation is relatively large. Li et al.[6] proposed a method to identify eye state by using the combination of two value image horizontal projection method and the Hough transform to find the circle. But this method is easy to be affected by the change of head angle, and the precision is not high.

In this paper, a fast algorithm for eye location and state detection is proposed, which is applicable to the case when the driver's head has a certain angle.

\section{Overview of System and Image Preprocessing}

The system consists five parts: image pre-processing, face detection, extraction the region of interest, eye location and state analysis. The flow chart is shown as below:

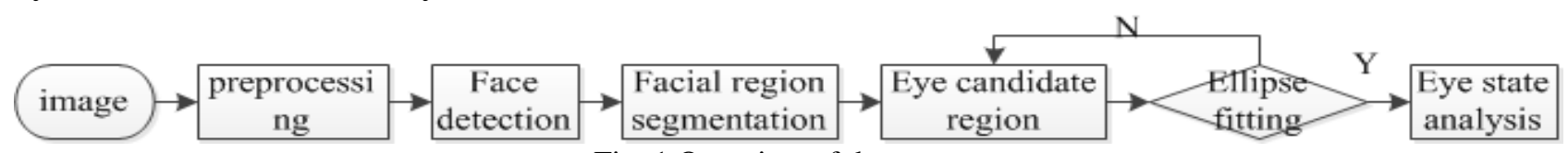

Fig. 1 Overview of the system

Due to the special nature of the car's inside environment, the collected images are often caused by uneven illumination. In order to improve the performance of the image analysis, in this system, histogram equalization is firstly used to improve image quality.

The pixel's gray levels are transferred from $\mathrm{r}_{\mathrm{k}}$ to $\mathrm{s}_{\mathrm{k}}$ according to the equation (1): 


$$
\mathrm{S}_{\mathrm{k}}=\mathrm{T}\left(\mathrm{r}_{\mathrm{k}}\right)=(\mathrm{L}-1) \sum_{\mathrm{j}=1}^{\mathrm{k}} \mathrm{p}_{\mathrm{r}}\left(\mathrm{r}_{\mathrm{j}}\right)=\frac{(\mathrm{L}-1)}{\mathbb{M N}} \sum_{\mathrm{j}=0}^{\mathrm{k}} \mathrm{n}_{\mathrm{j}}
$$

Wherein, $\mathrm{MN}$ is the total number of pixels in image; $\mathrm{L}$ means the number of gray levels; $\mathrm{n}_{\mathrm{j}}$ is the number of pixels which gray level is $\mathrm{r}_{\mathrm{k}}$.

\section{Face Detection and Facial Region Segmentation}

\subsection{Face Detection}

Face detection based on skin color model, which calculation is relatively small, can achieve face detection at faster speed. Besides, it is not influenced by the direction of the head or facial expression. So, we choose this algorithm for face detection.

Since YCbCr color space can better reflect the color clustering characteristics, so we convert the image into YCbCr space at first. Then, according to the theory presented by G.Kukharev, and A.Novosielsk[7], if $\mathrm{Y}, \mathrm{Cb}$, Cr values satisfy $\mathrm{Y}>80,85<\mathrm{Cr}<135,135<\mathrm{Cb}<180$, it can be considered belong to skin area. The result is as follows:

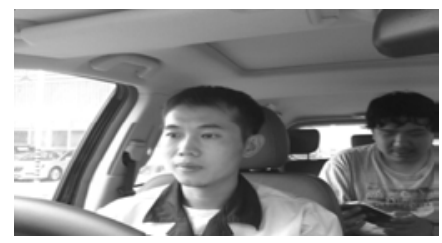

(a) Gray scale image

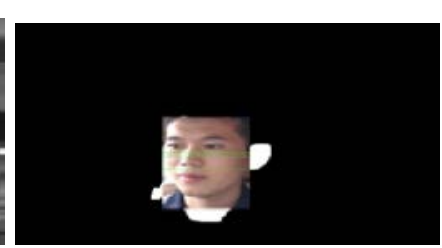

(b) facial region

\subsection{Ear Detection}

Fig. 2 Result of Face detection

After the completion of the human face location, the projection of a certain number of columns on the right side of the facial connected domain is done (the green box section in fig.3 (a)).Calculating the number of white pixels per column in this area. If the number of pixels meets specific properties, and 1) the total number of suspected ear is greater than $0.3 \mathrm{X}$ (X's value is the width of the face region); 2 ) the projection is continuous distribution. Then the image is considered to has an ear component:

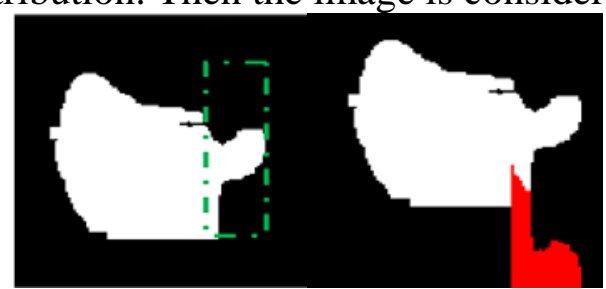

(a)facial edge (b) Projection result

\subsection{Mouth Detection}

Fig. 3 Result of right ear detection

As one of the practical methods, in the YIQ color domain, especially with Q-component, is adopted to detect mouth. Because Q-component can describe the independent saturation range from yellow, green to purple, a better performance is shown by Q-component than R-component in RGB domain. The conversion formula from RGB to YIQ is shown as below:

$$
\left[\begin{array}{l}
\mathrm{Y} \\
\mathrm{I} \\
\mathrm{Q}
\end{array}\right]=\left[\begin{array}{ccc}
0.299 & 0.587 & 0.114 \\
0.596 & -0.275 & -0.321 \\
0.212 & -0.523 & 0.311
\end{array}\right]\left[\begin{array}{l}
\mathrm{R} \\
\mathrm{G} \\
\mathrm{B}
\end{array}\right]
$$

Then, through a large number of experiment tests, we found that choosing $70 \%$ of maximum as the threshold parameter is sensible in mouth detection.

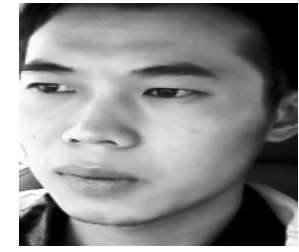

(a) facial image

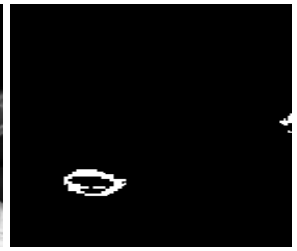

(b) mouth detection Fig. 4 Result of mouth detection 


\subsection{Facial Region Segmentation}

After locating of the mouth and the ear, then, we can divide the face image into four parts.

The symmetrical axis of the face is the straight line that is perpendicular to the lips and passes through the center of the lips. We set the coordinates of the corners of the mouth are (X1, Y1), (X2, Y2) respectively. Then the slope of the lip is $k=(y 2-y 1) /(x 2-x 1)$,therefore the slope of the face symmetry axis is $1 / \mathrm{k}$, dividing the face into two parts. Similarly, through ear's location information, the face is divided into another two parts. The result is shown in figure5.

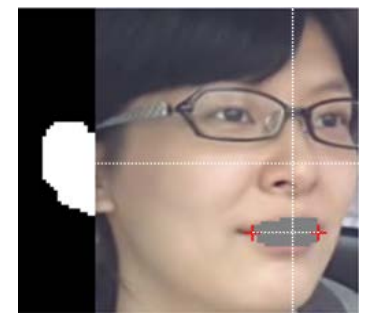

Fig. 5 Result of facial region segmentation

We notice that the right eye that is closer to the inside of the car is in the "upper left" part of the segmentation area. Here we think that, when driver is in driving process, his two eyes' state is same. Therefore, by detecting the state of the single eye, we can get the driver's state. The reason why choosing right eye is that it has a relatively clearer morphology compare to another one.

\section{Eye Location and State Analysis}

\subsection{Eye Selection}

The "upper left" region is extracted after segmentation, then using Otsu algorithm to covert the image to binary. By analysis each connected domain in this area, we can remove some connected domain that is definitely not the eye according to some basic morphological information. In the rest of the eye candidate connected domains, we further certify the right eye through the method of ellipse fitting.

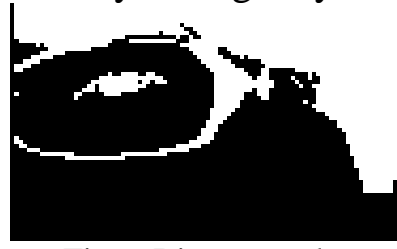

Fig. 6 Binary results

\subsection{Ellipse Fitting}

The shape of the eye is similar to an ellipse, so the accurate positioning of the eye among the candidate connected domains is realized by the ellipse fitting.

The any white spots can find the corresponding ellipse parameters by calculating its area 、 centroid position and second order moment. In the two value image, each pixel coordinates uses (R,C) to express. If there is a white spot in an area $\mathrm{R}$, then the area of the white spot can be expressed as

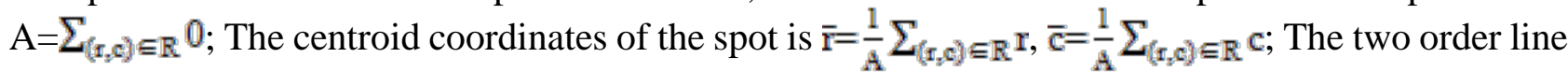
moment of the white spot is $\mathrm{u}_{\mathrm{rr}}=\frac{1}{\mathrm{~A}} \sum_{(\mathrm{r}, \mathrm{c}) \in \mathbb{R}}(\mathrm{r}-\overline{\mathrm{r}})^{2}$; Two order column moment is $\mathrm{u}_{\mathrm{cc}}=\frac{1}{\mathrm{~A}} \sum_{(\mathrm{r}, \mathrm{c}) \in \mathbb{R}}(\mathrm{c}-\overline{\mathrm{c}})^{2}$; Two order mixing moment is $\mathrm{u}_{\mathrm{rc}}=\frac{1}{\mathrm{~A}} \sum_{(\mathrm{r}, \mathrm{c}) \in \mathbb{R}}(\mathrm{c}-\overline{\mathrm{c}})(\mathrm{r}-\overline{\mathrm{r}})$.

The two axis of the ellipse can be expressed by second order matrix respectively:

$$
\begin{aligned}
& a=2 \sqrt{2} \sqrt{u_{u x}+u_{r x}+\sqrt{\left(u_{x c}-u_{u x}\right)^{2}+4 u_{u k}^{2}}} . \\
& \mathrm{b}=2 \sqrt{2} \sqrt{\mathrm{u}_{\mathrm{cx}}+\mathrm{u}_{\mathrm{ur}}-\sqrt{\left(\mathrm{u}_{\mathrm{wc}}-\mathrm{u}_{\mathrm{ur}}\right)^{2}+4 \mathrm{u}_{\mathrm{ul}}^{2}}} .
\end{aligned}
$$

According to the above formula we calculate the ellipse parameters corresponding to the white spot. When the white spot corresponds to an ellipse, there is the exact value of the ellipse parameters. 
However, when it not accord with an ellipse, then the result exist a certain deviation to the corresponding ellipse parameters. Here we defined ellipse hardness $\gamma$ to describe that deviation.

$$
\gamma=\frac{\pi \mathrm{sb}}{\mathrm{A}}
$$

That is the ratio of the elliptical area to the white spot area. The closer to 1 , the white spot is closer to an ellipse. The shape of the fitting ellipse of the eye pupil is shown in Figure 7.

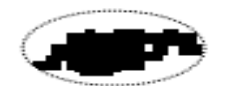

Fig. 7 Result of ellipse fitting

Through the experimental results, the eye can be determined by spot's ellipse properties, the rule is: The $\gamma$ is less than 1.5 , the ratio of the long short axis is less than 2.5 .

\subsection{Eye State Analysis}

Eye state is the opening degree of the eye, its most direct reflection is the size of the eye area. In the two value image, firstly, we can get white spot's (iris and pupil) area, called S'. Then its corresponding to the ellipse's area " $S$ " is:

$$
\mathrm{S}=\pi \mathrm{ab}
$$

Here, "a" ,"b" is the short, long axis of the ellipse respectively. Based on them, the opening degree of the eye can be expressed as $\mathrm{P}$ :

$$
\mathrm{P}=\frac{\mathrm{S}^{x}}{\mathrm{~S}}
$$

\section{Experiments and Results}

The following picture is the experimental environment.

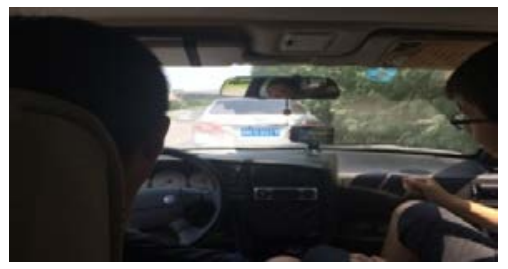

Fig. 8 Mobile phone position

All the experimental pictures used in this paper came from the actual vehicles. The testers are asked to simulate the driving state. The camera is placed on the platform inside the car, keeping a certain angle with the driver. In this paper, we collected 6000 images and each tester was asked to participate the experiment during different times of the day.

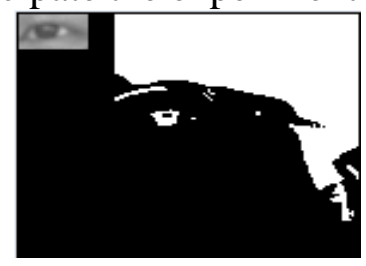

(a)tester1

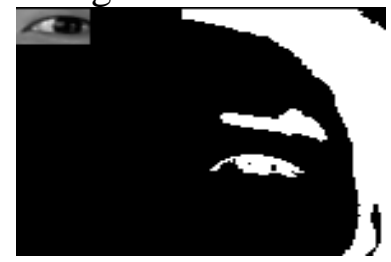

(b) tester2

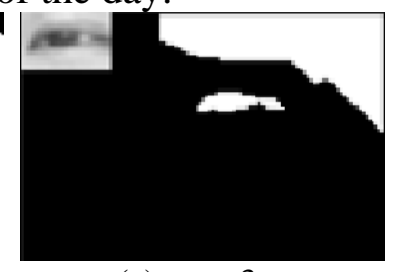

(c) tester3

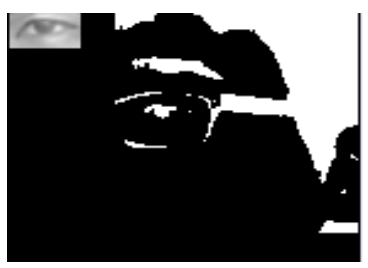

(d) tester4

Fig. 9 Result of eye location

The ellipse parameters of the eye in fig.9(c)(d) are shown in Table 1.In order to better compare with eyebrow region, the parameters of the fitting ellipse of the eyebrow region(fig.9(d))is also given in the table. As can be seen from table 1, the ellipse parameters corresponding to the eye meet the rule. 
Table 1 Human eye fitting ellipse parameters

\begin{tabular}{cccccc}
\hline \multirow{2}{*}{$\begin{array}{c}\text { Experiment } \\
\text { image }\end{array}$} & $\begin{array}{c}\text { Candidate } \\
\text { region }\end{array}$ & \multicolumn{4}{c}{ Ellipse parameter } \\
\cline { 3 - 6 } c & right eye & 14.2 & 6.4 & 1.2 & 2.2 \\
& right eyebrow & - & - & - & - \\
\hline \multirow{2}{*}{ d } & right eye & 7.4 & 4.8 & 1.3 & 1.5 \\
& right eyebrow & 24.5 & 5.9 & 1.7 & 4.2 \\
\hline \multirow{2}{*}{ "_" represents that there is no eyebrow in the region }
\end{tabular}

Table 2 shows the opening degree of the driver's eye in Figure 9. Generally speaking, $\mathrm{P}>70 \%$ belongs to the full open state; $30 \%<\mathrm{P}<70 \%$ belongs to half open; $\mathrm{P}<30 \%$ belongs to close state.

Table 2 Eye opening degree

\begin{tabular}{cc}
\hline Experiment image & Open degree(\%) \\
\hline $\mathrm{a}$ & 80 \\
$\mathrm{~b}$ & 87 \\
$\mathrm{c}$ & 83 \\
$\mathrm{~d}$ & 77
\end{tabular}

Table 3 gives a comparison of the eye location results by using different algorithms. Compared with them, the algorithm proposed in this paper not only need shorter time, but less affected by the change of the head angle. The accuracy is higher and has better robustness.

Table 3 Comparison with other algorithms

\begin{tabular}{ccccc}
\hline Method & $\begin{array}{c}\text { Video } \\
\text { (frame) }\end{array}$ & $\begin{array}{c}\text { Accuracy } \\
\text { (\%) }\end{array}$ & $\begin{array}{c}\text { Time } \\
\text { (s) }\end{array}$ & Results \\
\hline Gray level projection & 5000 & 76 & 14.2 & Inaccurate positioning \\
Template matching & 5000 & 90 & 58.4 & Large calculation \\
Symmetric transformation & 5000 & 72 & 25.6 & Effect is not good in side face \\
Method proposed in paper & 5000 & 94 & 19.4 & Fast, good positioning \\
\hline
\end{tabular}

\section{Conclusion}

In this paper, a fast eye detection and state judgment algorithm based on face region segmentation is proposed .First, the image is pre-processed. Then the driver's face region is located by using the skin color model method. By using the positional information of the mouth and ear organ, dividing the facial image, extracting the "upper left" part as region of interest. Finally, the method of ellipse fitting is used to realize the accurate positioning of the right eye and judge its state. Through the experiment, the algorithm is lesser influenced by the posture and the head angle of the driver. The algorithm is better, fast calculation speed and can be used in the designing of driver fatigue detection system.

\section{Acknowledgments}

This work was supported by National Natural Science Foundation of China (20222201 and 61305026) and Beijing Municipal Commission of Education (KM200710005009, KM201310005006 and PXM2009_014204_09_000154).

\section{References}

[1] Mark R. Rosekind Prepared Remarks. National Highway Traffic Safety Administration Lifesavers National Conference on Highway Safety Priorities on March 16, (2015)

[2] Sun W, Tang HQ. driver fatigue driving detection based on eye state[J].International Journal of Digital Content Technology and its Applications, (2011), p.307-314.

[3] Phimpltares S, Lursinsap C,. Face detection and facial feature localization without considering the appearance of image context [J]. Image and Vision Computing, (2007), p.741-753. 
[4] Huang Kun, HAN Fei, et al. Adaboost fast eye detection algorithm based on extended triangular features [J]. Journal of University of Science and Technology Beijing, (2012), p.48-52.

[5] Zhang Y, Zhao X Y, et al. A time delay neural network model for simulating eye gaze data [J], Journal of experimental and theoretical Artificial Intelligence, (2011), 23(1), p.111-126

[6] Li Y H, Zhu S A. Hough transfer for eye feature extraction [J]. Journal of Zhejiang University: engineering Science, 2008, 42(7), p.1164-1168.

[7] Kukharev.G, Nowosielski.N. Visitor identification elaborating real time face recognition system, WSCG Short Communication Papers proceedings, (2004), p.57-165. 